Trauma Surgery \& Acute Care Open

\title{
Using a hybrid approach to management of a common femoral arterial dissection
}

\author{
Hiromu Maehara, ${ }^{1}$ Taketo Kurozumi, ${ }^{2}$ Hiroshi Kondo, ${ }^{3}$ Tetsuya Sakamoto, ${ }^{1}$ Kaori Ito (i)
}

'Division of Acute Care Surgery, Department of Emergency Medicine, Teikyo University School of Medicine, Itabashi-ku, Tokyo, Japan

${ }^{2}$ Trauma and Reconstruction Center, Teikyo University Hospital, Itabashi-ku, Tokyo, Japan

${ }^{3}$ Department of Radiology, Teikyo University School of Medicine, Itabashi-ku, Tokyo, Japan

\section{Correspondence to} Dr Kaori Ito; kaoriito1@gmail. com

\section{WHAT WOULD YOU DO?}

A middle-aged (fifth decade) patient was brought to the emergency department after a motorcycle collision during which the lower abdomen-to-pelvis area was trapped between a traffic sign pole and the motorcycle. On admission, vital signs were normal. The patient complained of left groin and buttock pain. Primary and secondary evaluations revealed subcutaneous hematoma and tenderness in the groin bilaterally and left buttock. Left dorsal pedis arterial pulse was slightly diminished compared with that on the right. No neurological deficits were apparent in bilateral lower extremities (LEs). Laboratory test results were unremarkable. Contrast-enhanced CT revealed a defect in the left common femoral artery (CFA) with normal runoff. Scans also identified a sacral bone fracture with contrast medium extravasation and a pseudoaneurysm with a large subcutaneous hematoma in the left gluteus maximus muscle (figure 1A,B).

As the vascular surgery, interventional radiology, and orthopedic departments were planning treatment, the patient, now tachycardic and diaphoretic, complained of worsening pain and numbness in the left buttock and LEs. Examination showed that the subcutaneous hematoma in its left gluteal region had expanded significantly and was firmer. The left LE was cold and pale, with no palpable pulses or Doppler signals. Left ankle dorsal flexion was impaired. These neurological deficits were compatible with sciatic nerve palsy and ischemia. On palpation, left LE muscles were soft and tender. Since admission, the hemoglobin level decreased from 12.1 to $10.2 \mathrm{~g} / \mathrm{dL}$, and lactic acid level increased from 2.0 to $5.9 \mathrm{mmol} / \mathrm{L}$. We suspected left LE ischemia secondary to left CFA occlusion and left gluteal compartment syndrome causing left sciatic nerve palsy due to the expanding gluteal hematoma and active arterial hemorrhage.

\section{WHAT WE DID AND WHY}

We first performed angiography, accessing the right CFA and placing an introducer. Aortography revealed left CFA flow defects. Blood flow was intact distal to the superficial-deep femoral artery's bifurcation. Contrast medium extravasation was seen from the left inferior gluteal artery into the left gluteal hematoma. Because the dissection was focal (within $2 \mathrm{~cm}$ ), we introduced a percutaneous transcatheter angioplasty (PTA) balloon $(8-10 \mathrm{~mm}$ diameter, $4 \mathrm{~cm}$ long) over a guide wire into the CFA and insufflated it to expand the vessel, where it remained for $20 \mathrm{~s}$, three times. Intravenous heparin (5000 U) was used. Flow was restored in the CFA

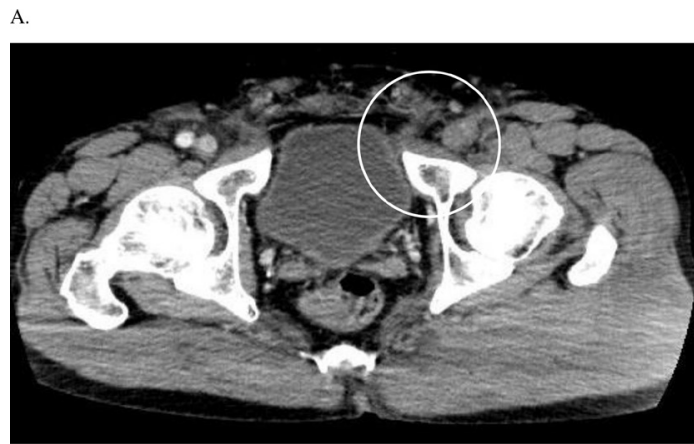

B.

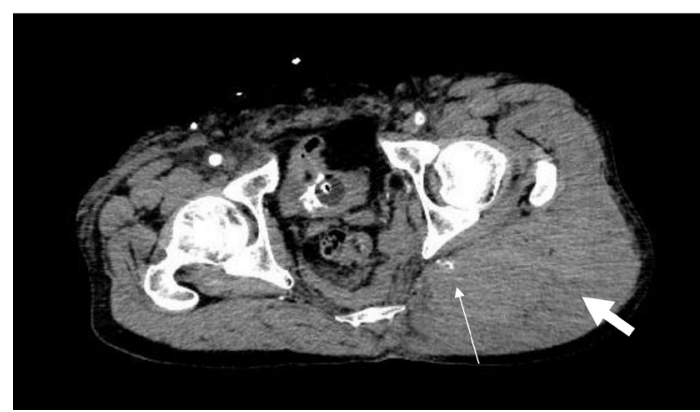

Figure 1 (A) Contrast-enhanced CT shows that the left common femoral artery is not visible (circle). (B) Gluteal hematoma (thick arrow) with extravasation of contrast medium from the left inferior gluteal artery (thin arrow).

with distal runoff (figure 2A,B). Ankle-brachial index was 0.75 . The left gluteal artery then underwent transcatheter arterial embolization.

Following embolization, the patient was placed in right lateral position. Orthopedic surgeons evacuated the left gluteal hematoma, removing approximately $500 \mathrm{~g}$ clotted blood, which relieved compression of the left sciatic nerve. The wound was closed after confirming hemostasis. The patient was then placed in supine position, and vascular surgeons repaired the left CFA with an interpositional prosthetic graft. The resected left CFA segment $(2.0 \mathrm{~cm})$ had sustained intimal injury with flap formation (figure 3 ).

Postoperatively, the left LE was well perfused, gradually resolving the paresthesia and paralysis. Postoperative hospital course was unremarkable except for lymphocele formation around the CFA graft, which was treated with repeated percutaneous aspiration. Patient was discharged home on hospital day 73 without disabilities.

This case was challenging because of two simultaneous, critical problems: LE ischemia secondary to CFA occlusion and gluteal compartment syndrome due to the expanding hematoma. Hemorrhage 
A.

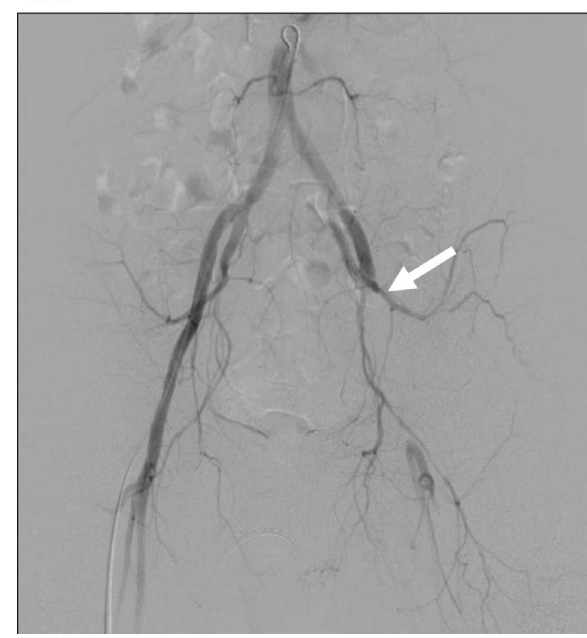

B.

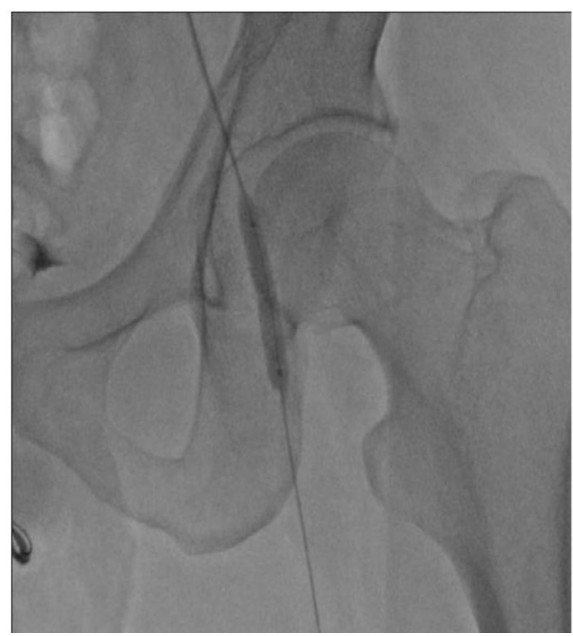

C.

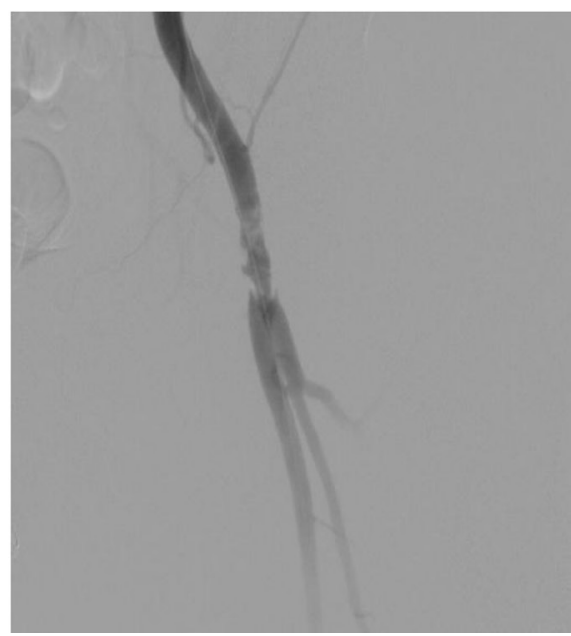

Figure 2 (A) Aortography shows interrupted blood flow in the left common femoral artery (CFA). (B) The percutaneous transcatheter angioplasty (PTA) balloon was deployed in the occluded left CFA. (C) Blood flow is restored distal to the CFA after PTA.

control and immediate restoration of LE perfusion were priorities. We used an endovascular approach to achieve hemostasis of the gluteal vessels and restoration of CFA flow. Orthopedic surgeons then addressed the gluteal compartment syndrome without perfusion in the LE, and vascular surgeons made definitive repairs without gluteal hemorrhage or compartment syndrome concerns.

There is increasing recognition of the utility of endovascular stenting for blunt peripheral vascular injuries. Most reported cases have focused on blunt injuries of the axillary artery. Blunt CFA injury is not generally considered for endovascular treatment. The few studies describing endovascular stenting for blunt

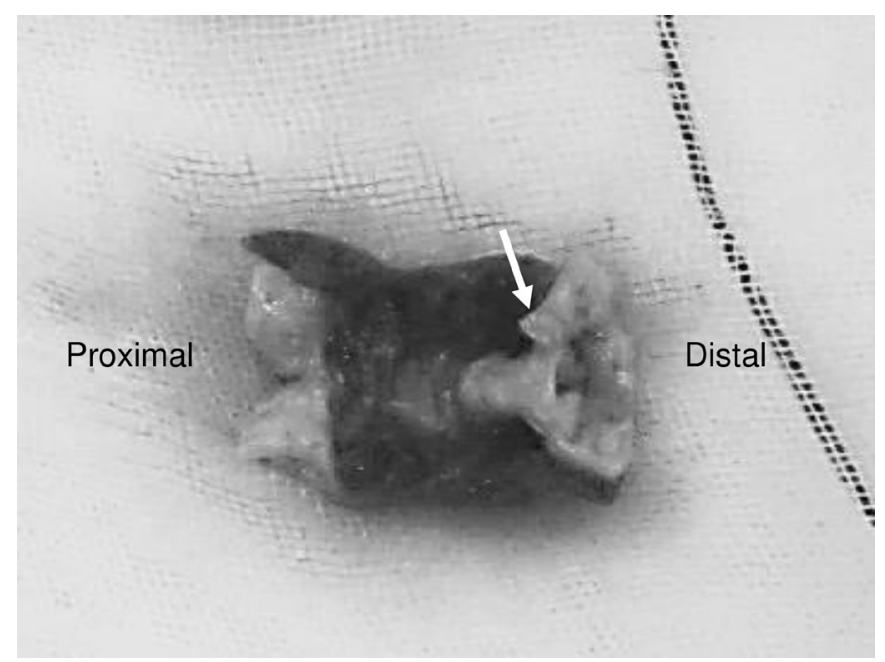

Figure 3 Specimen from the resected common femoral artery. The intima is inverted and forms a flap.
CFA injury have not clarified its feasibility. Emergence of the hybrid operating room is accelerating the use of endovascular treatment for polytrauma patients as it enables endovascular and open surgery simultaneously/subsequently. Our patient would have been a good candidate for a hybrid operating room had it been available. We found no previous reports of using PTA balloon dilatation to salvage blood flow distal to the CFA prior to definitive treatment. Of note, this technique is viable only when immediate open surgical repair or shunting of the CFA is impossible (eg, when other problems with higher priority exist (eg, active bleeding) must be addressed, no available surgeon for the definitive repair). Because blunt vascular injury involves intimal injury accompanied by flap formation, the injured arterial segment must be resected and reconstructed even after PTA salvage.

Contributors Conception and design: HM, KI. Acquisition of data: HM. Analysis and interpretation of data: N/A. Drafting of the manuscript: HM, KI. Critical revision of the manuscript: HK, TK, TS. Statistical expertise: N/A. Supervision: TS, KI.

Funding The authors have not declared a specific grant for this research from any funding agency in the public, commercial or not-for-profit sectors.

Competing interests None declared.

Patient consent for publication Obtained.

Ethics approval The study obtained ethics approval by Teikyo University Institutional Review Board (TUIC-COI18-0860).

Provenance and peer review Not commissioned; externally peer reviewed.

Open access This is an open access article distributed in accordance with the Creative Commons Attribution Non Commercial (CC BY-NC 4.0) license, which permits others to distribute, remix, adapt, build upon this work non-commercially, and license their derivative works on different terms, provided the original work is properly cited, appropriate credit is given, any changes made indicated, and the use is non-commercial. See: http://creativecommons.org/licenses/by-nc/4.0/.

\section{ORCID iD}

Kaori Ito http://orcid.org/0000-0001-5276-2064 